\title{
ACCELERATED WOUND HEALING ABILITY OF SACRAN HYDROGEL FILM BY KERATINOCYTE GROWTH FACTOR IN ALLOXAN-INDUCED DIABETIC MICE
}

\author{
NASRUL WATHONI ${ }^{1,3^{*}}$, ALIYA NUR HASANAH², AHMED FOUAD ABDELWAHAB MOHAMMED ${ }^{3,4}$, ELASARI DWI \\ PRATIWI $^{1}$, RIPA'ATUL MAHMUDAH ${ }^{1}$
}

1Department of Pharmaceutics, Faculty of Pharmacy, Universitas Padjadjaran, Jatinangor 45363, Indonesia, ${ }^{2}$ Department of Analytical Pharmacy and Medicinal Chemistry, Faculty of Pharmacy, Universitas Padjadjaran, Jatinangor 45363, Indonesia, ${ }^{3}$ Graduate School of Pharmaceutical Sciences, Kumamoto University, Kumamoto 8620973, Japan, 4 Faculty of Pharmacy, Minia University, Minia 61517, Egypt Email: nasrul@unpad.ac.id

Received: 10 Dec 2017, Revised and Accepted: 23 Jan 2018

\section{ABSTRACT}

Objective: The main objective of the research work was to fabricate sacran hydrogel film containing keratinocyte growth factor (Sacran/KGF-HGF), and to evaluate their wound healing ability in alloxan-induced diabetic mice model.

Methods: The physicochemical characterization of Sacran/KGF-HGF were investigated by thickness, tensile strength, swelling ratio, $\mathrm{x}$-ray diffractometer (XRD), scanning electron microscope (SEM), and biodegradability. The wound healing ability was investigated by creating two fullthickness excisional wounds inalloxan-induced diabetic mice.

Results: The thickness, tensile strength, and swelling ratio results showed that KGF in the Sacran/KGF-HGF improved not only the thickness of sacran hydrogel film (Sacran-HGF), but also the tensile strength and swelling ability of Sacran-HGF. The XRD and SEM results confirmed that the Sacran/KGF-HGF were amorphous and similar morphology to Sacran-HGF, respectively. The biodegradability results revealed that the Sacran/KGF-HGF degraded for about $41.29 \%$ in trichloroacetic acid (TCA) and $22.92 \%$ in TrypLE ${ }^{\text {TM }}$ (recombinant enzyme) solutions. In addition, KGF improved the degradability of Sacran/KGF-HGF in both solutions. Interestingly, the Sacran/KGF-HGF, which was applied on wound site, considerably improved the wound healing ability of Sacran-HGF at 6, 9 and $12 \mathrm{~d}$ in alloxan-induced diabetic mice model, compared to control (non-treated).

Conclusion: These results suggest that KGF has the potential to promote the chronic wound healing ability of Sacran-HGF.

Keywords: Sacran, Hydrogel film, Keratinocyte growth factor, Wound healing

(C) 2018 The Authors. Published by Innovare Academic Sciences Pvt Ltd. This is an open access article under the CC BY license (http://creativecommons.org/licenses/by/4.0/) DOI: http://dx.doi.org/10.22159/ijap.2018v10i2.24217

\section{INTRODUCTION}

The skin, a layered organ, covers the entire human body with the main function as a protector for preventing skin dehydration and microorganism's penetration from outside the body. If the skin is injured, it provides complex natural biological reactions and complicated wound healing processes [1]. The wound healing process basically consists of several stages, namely hemostasis, inflammation, proliferation, vascularization, production and restoration of the extracellular matrix (ECM)[2]. However, the healing process in chronic wounds, especially in diabetic condition, will be disrupted with persistent inflammation, slow migration of keratinocytes and fibroblast, abnormal regulation of chemokines and production of growth factor, the irregular response of inflammatory cells, and inhibition of angiogenesis [3-5]. Recently, a number of studies using new therapy for diabetic wound infections were investigated [6, 7]. Regenerative therapy using growth factors can be used to accelerate the chronic wounds healing $[8,9]$. One of the essential growth factors for impaired healing therapy is Keratinocyte Growth Factor (KGF). KGF is a major cellular component of the epidermis, not only important for the maintenance of the barrier, but also for the restoration of the wound through a process known as epithelization [10]. KGF also acts specifically on epithelial cells and stimulates cell proliferation and migration [11].

Nowadays, the concept of wound healing by maintaining a moist environment at the wound site has been established as a standard therapy for chronic wounds [12, 13]. The moist wound environments can accelerate the wound healing process by enhance re-epithelization, stimulate collagen synthesis, increase growth factor activity, and accelerate the healing process [14].

Recently, the modern wound dressings have been developed to preserve and protect a moist environment in the both acute and chronic wounds [15]. The dressings are available in the forms of films, foam, hydrogel and hydrocolloids, which can be incorporated with a bioactive agent to promote wound healing [16]. Among other forms, the thin hydrogel films (HGFs) consisting of water-swollen polymer networks have attracted a lot of attention for the last few decades due to their excellent properties such as adhesive, porous, semi-permeable, flexible and easily conformable, for the treatment of acute and chronic wounds [17].

During last two decades, natural polymers are widely used in the regenerative medicine field, outstandingly for wounds dressing because of their biocompatibility, biodegradability and similarity to the extracellular matrix [18]. Natural polymers are involved in the repair of damaged tissues and consequently in skin regeneration, inducing and stimulating the wound healing process [19]. In addition, the swollen ability of natural polymer-based hydrogels has some functions to absorb the overage of wound exudate and accelerate autolysis process for wound debridement [20].

Sacran, a giant molecular polysaccharide, was newly isolated from cyanobacterium Aphanothece sacrum. Sacran has the unique properties to be developed as a new biomaterial in HGFs for wound dressing application, such as a very high molecular weight $\left(2.9 \times 10^{7} \mathrm{Da}\right)$, a safe biomaterial and a high moisturizing effect [21]. In the previous study, we successfully developed a physically crosslinked Sacran-HGF and revealed its potential as a novel wound dressing material [22]. Furthermore, we confirmed that Sacran-HGF has the potential to deliver water-soluble complex of curcumin and 2-hydroxypropyl- $\gamma$-cyclodextrin at the wound site and promote the acute wound healing ability [23, 24]. Until now, there is no study regarding the combination of KGF with sacran HGF (Sacran/KGF-HGF) for chronic wound therapy. Therefore, we fabricated Sacran/KGF-HGF, then evaluated their physicochemical properties and wound healing ability in diabetic mice model. 


\section{MATERIALS AND METHODS}

\section{Materials}

The sacran was kindly provided by Green Science Material (Kumamoto, Japan). Keratonicyte growth factor (KGF) was purchased from Skin Actives Scientific (Arizona, USA). Trichloroacetic acid (TCA) and TrypLE ${ }^{\mathrm{TM}}$ (recombinant enzyme) were bought from Wako Chemical (Tokyo, Japan). All the reagents were analytical grade and used without further purification.

\section{Preparation of sacran/KGF-HGF}

The preparation method of the Sacran-HGF was briefly described in our previous study using a solvent evaporation method[22]. The Sacran-HGF $\left(1 \times 1 \mathrm{~cm}^{2}\right)$ was placed in Tissue-TekCryomold ${ }^{\circledR}$ (Sakura Finetek, Tokyo, Japan). Then, $10 \mu \mathrm{L}$ of KGFs (100 ng) in ammonium sulfate solution were placed and casted on the surface of the SacranHGF. The Sacran-HGF was swollen for $24 \mathrm{~h}$ at $4{ }^{\circ} \mathrm{C}$ with light protection and dried for $4 \mathrm{~h}$ at $37^{\circ} \mathrm{C}$ to obtain the Sacran/KGF-HGFs.

\section{Thickness test of sacran/KGF-HGF}

A dial thickness gauge (Teclock Corp., Nagano, Japan) was used to measure the thickness of Sacran-HGFs.

\section{Tensile strength of sacran/KGF-HGF}

The sacran/KGF-HGFs were clipped with 2 holding grips and pulled by the top clip at a rate of $0.5 \mathrm{~mm} / \mathrm{s}$ in Textechnofavigraph (Moenchengladbach, Germany). Tensile strength was measured from the breakpoint of the HGFs for 3 times, then, the average value was calculated by tensile strength equation.

$$
\mathrm{MPa}=\frac{\mathrm{p}}{\mathrm{bxd}}
$$

( $\mathrm{p}$ = breakpoint, $\mathrm{b}=$ width, $\mathrm{d}=$ thickness)

\section{Swollen ratio of sacran/KGF-HGF}

The sacran/KGF-HGFs $\left(1 \times 1 \mathrm{~cm}^{2}\right)$ were immersed in PBS (pH 7.4) for $24 \mathrm{~h}$ and weighted $\left(\mathrm{W}_{\mathrm{t}}\right)$ at room temperature. The gravimetric method was used to determine a swollen ratio of the sacran/KGFHGFs [25]. It was measured by comparing $\mathrm{W}_{\mathrm{t}}$ and initial weight $\left(\mathrm{W}_{\mathrm{o}}\right)$, and calculated from the following equation [26].

$$
\mathrm{q}=\frac{\left(\mathrm{Wt}-\mathrm{Wo}_{\mathrm{o}}\right)}{\mathrm{Wo}_{\mathrm{O}}}
$$

\section{Scanning electron microscopy analysis}

The sacran/KGF-HGFs were coated with gold for $10 \mathrm{~s}$ using the JFC1200 Fine Coater (JEOL, Tokyo, Japan). The surface of the HGFs was analyzed using scanning electron microscopy (SEM) with a JSM6510LA (JEOL, Tokyo, Japan) in $5 \mu \mathrm{l}$ (x3000).

\section{$\mathrm{X}$-ray diffractometric analysis}

The x-ray diffraction (XRD) patterns were acquired by a Powder Xray diffractometer (Rigaku Ultima IV, Tokyo, Japan). Briefly, the Sacran/KGF-HGFs were kept on a sample holder. The XRD settings were as follows: a Ni-filtered $\mathrm{Cu}-\mathrm{K} \alpha$ radiation, the voltage of $40 \mathrm{kV}$, a current of $20 \mathrm{~mA}$, divergent slit of $10 \mathrm{~mm}\left(0.5^{\circ}\right)$, a scanning speed of $5 \% \mathrm{~min}$, opened scattering and receiving slits.

\section{Biodegradability of sacran/KGF-HGF}

The sacran/KGF-HGFs $\left(1 \times 1 \mathrm{~cm}^{2}\right)$ were weighed $\left(\mathrm{W}_{\mathrm{o}}\right)$ and added with $2 \mathrm{ml}$ TCA and TrypLE' ${ }^{\mathrm{TM}}(20 \%)$ solutions for $24 \mathrm{~h}$. Then, HGFs were weighed as the final weight $\left(\mathrm{W}_{\mathrm{t}}\right)$ and calculated the percent change in weight by the formula, Percent in Weight $=100$ $\left(\mathrm{W}_{\mathrm{t}} / \mathrm{W}_{\mathrm{o}} * 100\right)$.

\section{Alloxan-induced diabetic mice}

Male albino mice (strain: BALB/c, age: 6-8 w, body weight: 30-35 g, source: laboratory of pharmacology, teaching hospital, Universitas Padjadjaran) were housed under standard conditions of control temperature and humidity $\left(25^{\circ} \mathrm{C} \pm 1,40 \%\right)$ and a light/dark cycle
$(12 / 12 \mathrm{~h})$. Before diabetic induction by alloxan, the mice fasted for 8 h. Alloxan monohydrate $(186.9 \mathrm{mg} / \mathrm{kg}$ body weight) was injected by intraperitoneal injection. $1 \mathrm{~h}$ after induction, $5 \%$ of glucose solution was applied to the mice for $24 \mathrm{~h}$, then, replaced by distilled water. The blood glucose level of mice was measured using glucometer after $3 \mathrm{~d}$ of induction. Alloxan-induced diabetic mice had blood glucose levels at $150-250 \mathrm{mg} / \mathrm{dl}$ on the 3rd day after alloxan induction.

\section{Wound closure study of sacran/KGF-HGF}

The in vivo wound closure studies were performed as reported previous [22]. Briefly, two full-thickness excisional wounds were made on the dorsal side of hairless mice using 8-mm biopsy punch tools. Three mouse groups were prepared as Sacran-HGF, Sacran/KGF-HGFand control (without treatment). The HGFs were applied precisely on the wound site, after 6,9 and $12 \mathrm{~d}$, the wound areas were digitally photographed and quantified by Image J software. The wound sizes were expressed as a percentage of the respective initial wounds. All the animal research studies were approved by the Ethics Committee for Animal Care and Use of Kumamoto University (Ethical approval number: 25-203).

\section{Statistical analysis}

The quantitative data were expressed as the mean \pm standard error of the mean (SEM) while the statistical comparisons were made using the Scheffe's test. A $p$-value $<0.05$ was considered statistically significant.

\section{RESULTS AND DISCUSSION}

\section{Preparation of sacran/KGF-HGF}

In the previous study, we successfully prepared a physically crosslinked Sacran-HGF using the solvent-evaporation method and considerably improved acute wound healing ability [22]. In this study, we next examined whether Sacran-HGFs can be formed in the presence of KGF for chronic wound therapy, and evaluated their physicochemical properties. Sacran/KGF-HGF was fabricated with the thickness of the film was higher then that of sacran-HGF (fig. 1ab). These results suggest that the KGF was effectively incorporated in the sacran-HGF.

\section{Physicochemical properties of sacran/KGF-HGF}

The HGFs need an excellent mechanical property for wound dressing application [27]. Therefore, we examined the tensile strength of sacran/KGF-HGF compared to sacran-HGF without KGF. The tensile strength of sacran/KGF-HGFs was determined from the breakpoint of the HGFs in the Textechnofavigraph apparatus. Fig. 1c showed that the addition of KGF in sacran-HGF significantly increased the tensile strength of sacran/KGF-HGF for 1.5 fold of Sacran-HGF (4 $\mathrm{Mpa})$, indicating that sacran/KGF-HGF stronger then that of sacranHGF.

One of the ideal properties of the HGFs is a high swelling ability which is connected to the moisturizing effect of HGFs [28]. Therefore, we next evaluated the swelling ability of the sacran/KGFHGF. The gravimetric method was used to calculate the swelling ratio of the HGFs after immersing in PBS for $24 \mathrm{~h}$, comparing to the initial weight. As shown in fig. 1d, the sacran/KGF-HGF was swollen about 12 -fold compared to the initial weight. Meanwhile, sacranHGF was swollen about 10 -fold compared to the initial weight. In the previous study, the dried film of cross-linked hyaluronic acid hydrogel films swelled sevenfold in volume [29]. These results suggest that the sacran/KGF-HGF improved the swelling ability of sacran-HGF and it is higher then that of cross-linked hyaluronic acid hydrogel films.

To understand the morphology of the sacran/KGF-HGF, we investigated the HGFs by SEM. The surface of the HGFs was analyzed in $5 \mu \mathrm{l}(\mathrm{x} 3000)$. It can be seen in fig. 2, the appearances of KGF could not be detected on the surface of Sacran-HGF, suggesting the KGF penetrate inside the HGFs. Further investigation is needed to clarify these findings. 
(a)

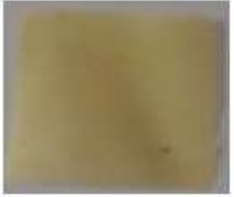

Sacran-HGF

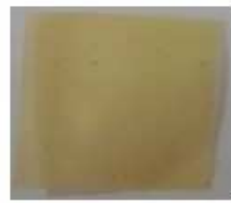

Sacran/KGF-HGF

(b)

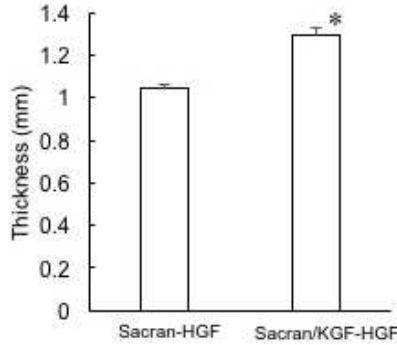

(c)

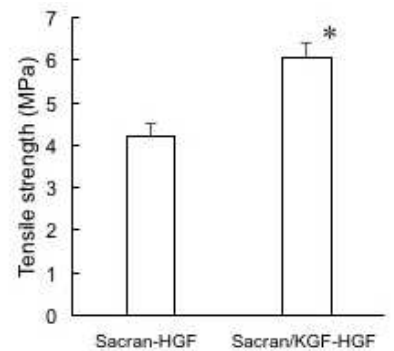

(d)

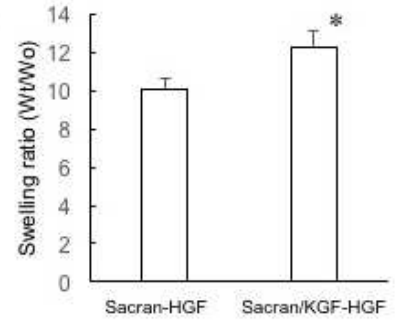

Fig. 1: Physicochemical properties of sacran/KGF-HGF (a) Appearances (b) Thickness (c) Tensile strength (d) Swelling ratio. Each value represents the mean $\pm S$. E. of 3 experiments. ${ }^{*} p<0.05$ compared to sacran-HGF

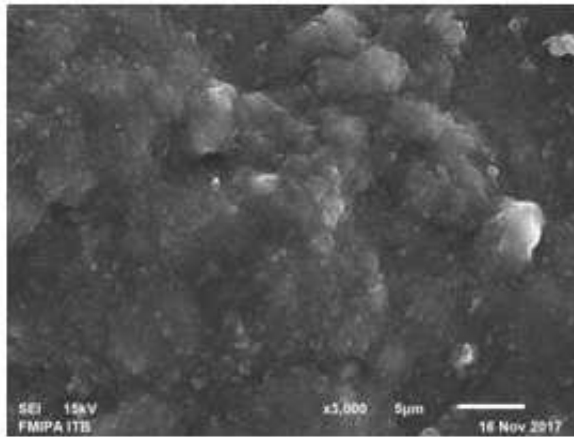

Sacran-HGF

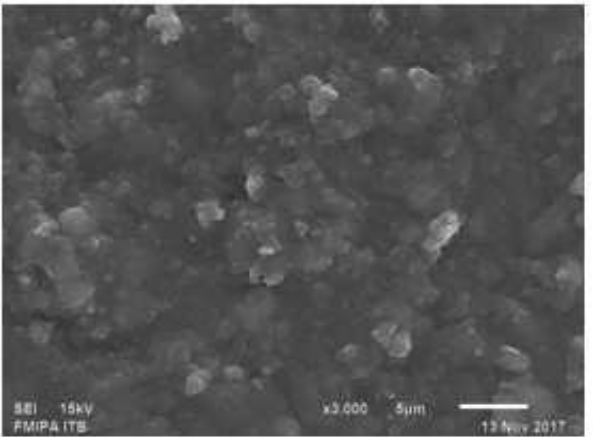

Sacran/KGF-HGF

Fig. 2: Scanning electron microscopy analysis of sacran/KGF-HGF, an amorphous HGFs is important due to a high thermodynamic system and a high solubility [24]. To clarify the amorphous form in the sacran/KGF-HGF, we used an x-ray diffractometer. The results showed that the sacran/KGF-HGF had hallow pattern as same as sacran-HGF, indicating a high thermodynamic activities (fig. 3)
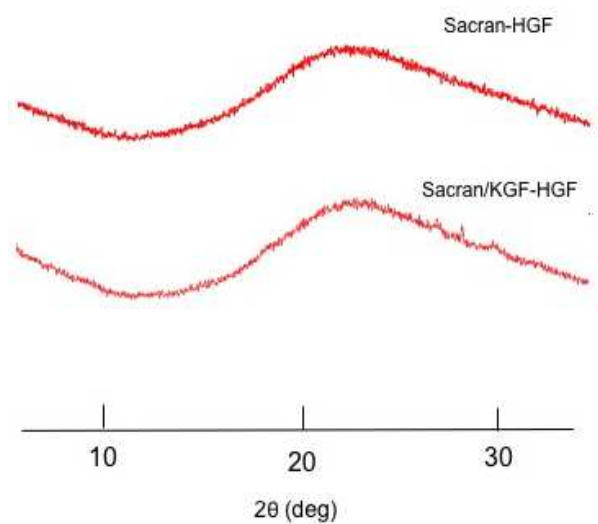

Fig. 3: X-Ray diffractometer pattern of sacran/KGF-HGF

\section{Biodegradability of sacran/KGF-HGF}

HGFs for wound dressing application should be biodegradable and presents a suitable environment for the tissue repair $[30,31]$. Therefore, we next evaluated the biodegradability of sacran/KGFHGF in chemical reagent (TCA) and enzyme (TrypLE ${ }^{\text {TM }}$ ) solutions.
Fig. 4 showed that sacran/KGF-HGF degraded for about $41.29 \%$ in TCA and $22.92 \%$ in the enzyme. In addition, KGF improved the degradability of sacran/KGF-HGF in both solutions. These results suggest that KGF has a synergistic effect with Sacran-HGF for biodegradability of HGF.

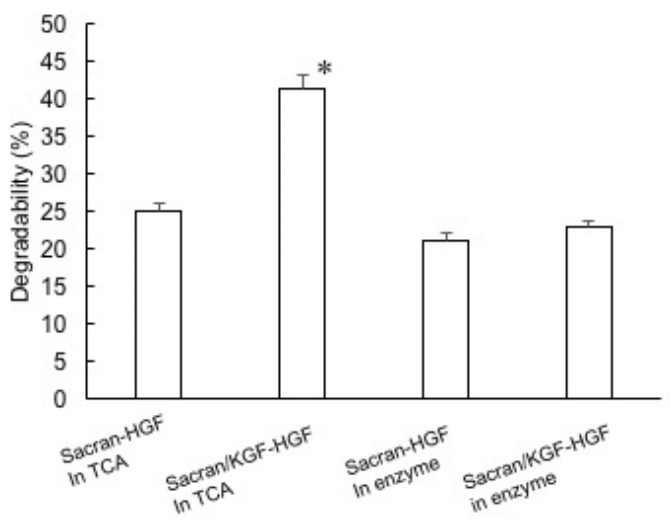

Fig. 4: Biodegradability of sacran/KGF-HGF. Each value represents the mean \pm SE of 3 experiments. ${ }^{*} p<0.05$ compared to sacran-HGF in TCA 


\section{Wound closure study}

Finally, to clarify the effect of KGF in sacran-HGF for chronic wound healing, we created an alloxan-induced diabetic model for wound closure study. A higher dose of alloxan ( $180 \mathrm{mg} / \mathrm{kg}$ body weight) in mice can be used to create severe atrophy of pancreatic islets and induced pancreatic $\beta$ cell apoptosis [32]. We prepared three mouse groups of sacran-HGF, Sacran/KGF-HGF and control (without treatment). Then, the HGFs were applied precisely on the wound site at $0 \mathrm{~d}$. After 6,9 and $12 \mathrm{~d}$, the wound areas were digitally photographed and quantified by Image J software (fig. 5a-b). The sacran/KGF-HGF significantly accelerated the wound healing ability of sacran-HGF at 6, 9 and $12 \mathrm{~d}$, indicating there is a positive effect between KGF and sacran-HGF in wound healing ability. Jimenej and Rampy confirmed that treatment of KGF resulted in an improvement in incisional wound healing due to an increase in breaking strength, collagen content, and epidermal thickness [33]. In addition, our previous study revealed that sacran-HGF increased wound healing ability, probably due to not only the moisturing effect but also the anti-inflammatory effect of sacran [22].
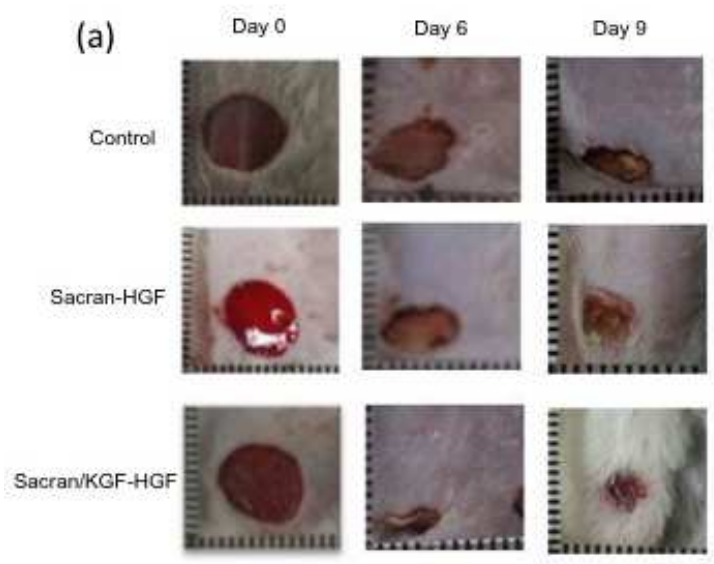

Day 12
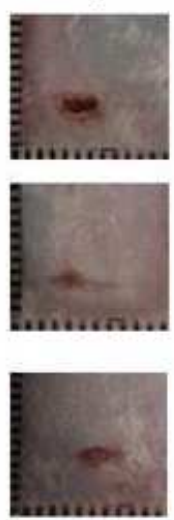

(b)

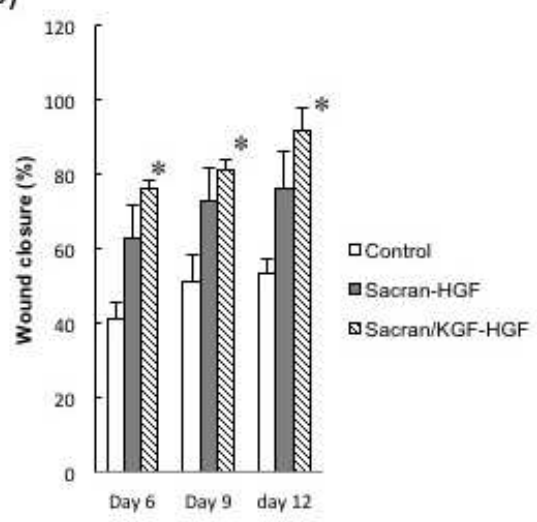

Fig. 5: Wound healing of sacran/KGF-HGF (a) Appearances (b) Wound Closure. Each value represents the mean \pm S. E. of 3 experiments. ${ }^{*} p<0.05$ compared to control

\section{CONCLUSION}

In this study, we successfully fabricated sacran/KGF-HGF for chronic wound healing with excellent physicochemical properties. The existence of KGF in sacran-HGF improved the thickness, swelling ability, and tensile strength of sacran-HGF. In addition, sacran/KGFHGF had an amorphous form and similar morphology to sacran-HGF. Moreover, KGF improved the degradability of sacran/KGF-HGF. Importantly, the sacran/KGF-HGF significantly accelerated the wound healing ability of sacran-HGF at 6, 9 and $12 \mathrm{~d}$ in alloxan-induced diabetic mice. These results suggest that KGF has the potential to promote the chronic wound healing ability of sacran-HGF.

\section{ACKNOWLEDGEMENT}

The authors thank Universitas Padjadjaran for providing research grant, and Mr. Shinichiro Kaneko, a CEO of Green Science Material, for providing sacran.

\section{AUTHORS CONTRIBUTIONS}

All the author have contributed equally

\section{CONFLICT OF INTERESTS}

The authors have no conflict of interest directly relevant to the content of this article.

\section{REFERENCES}

1. Broughton GII, Janis JE, Attinger CE. The basic science of wound healing. Plast Reconstr Surg 2006;117 Suppl 7:12-34.

2. Sorg H, Reinke JM. Wound repair and regeneration. Eur Surg Res 2012;49:35-43.

3. Martin A, Komada MR, Sane DC. Abnormal angiogenesis in diabetes mellitus. Med Res Rev 2003;23:117-45.

4. O'Loughlin A, Kulkarni M, Creane M, Vaughan EE, Mooney EG, Shaw, et al. Topical Administration of allogeneic mesenchymal stromal cells seeded in a collagen scaffold augments wound healing and increases angiogenesis in the diabetic rabbit ulcer. Diabetes 2013;62:2588-94.

5. Xu F, Zhang C, Graves DT. Abnormal cell responses and role of TNF-a in impaired diabetic wound healing. Biomed Res Int 2013:1-8. http://dx.doi.org/10.1155/2013/754802.
6. Khatri SK, Rathman and M, Nikhila R. Formulation and evaluation of wound healing activity of linezolid topical preparations on diabetic rats. Asian J Pharm Clin Res 2016;8:30-6.

7. Sushanth KA, Lakshmi KC, Reddy DS. Evaluation of wound healing activity with a new formulation of dry mangiferaindica and honey using swiss albino mice. Int J Appl Pharm 2016;9:139-42.

8. Joao De Masi ECD, Campos ACL, Joao De Masi FD, Ratti MAS, Ike IS, Joao De Masi RD. The influence of growth factors on skin wound healing in rats. Braz J Otorhinolaryngol 2016;82:512-21.

9. Ram M, Singh V, Kumawat S, Kant V, Tandan SK, Kumar D. Bilirubin modulated cytokines, growth factors and angiogenesis to improve cutaneous wound healing process in diabetic rats. Int Immunopharmacol 2016;30:137-49.

10. Pastar I, Stojadinovic O, Yin NC, Ramirez H, Nusbaum AG, Sawaya A, et al. Epithelialization in wound healing: a comprehensive review. Adv Wound Care (New Rochelle) 2014;3:445-64.

11. Werner S. Keratinocyte growth factor: a unique player in epithelial repair processes. Cytokine Growth Factor Rev 1998;2:153-65.

12. Margolis DJ, Hoffstad O, Nafash J, Leonard CE, Freeman CP, Hennessy S, et al. Location, location, location: geographic clustering of lower-extremity amputation among medicare beneficiaries with diabetes. Diabetes Care 2011;34:2363-7.

13. Ovington LG. Advances in wound dressings. Clin Dermatol 2007;25:33-8.

14. Martin C, Low WL, Amin MCIM, Radecka I, Raj P, Kenward K. Current trends in the development of wound dressings, biomaterials and devices. Pharm Pat Anal 2013;3:341-59.

15. Sarabahi S. Recent advances in topical wound care. Indian J Plast Surg 2012;45:379-87.

16. Schoukens S. Bioactive dressings to promote wound healing. Advanced Textiles for Wound Care, A volume in Woodhead Publishing Series in Textiles; 2009. p. 114-52.

17. Dhivya S, Padma VV, Santhini E. Wound dressings-a review. Biomedicine 2015;5:24-8.

18. Mogoşanu GD, Grumezescu AM. Natural and synthetic polymers for wounds and burns dressing. Int J Pharm 2014;463:127-36.

19. Ko HF, Sfeir C, Kumta PN. Novel synthesis strategies for natural polymer and composite biomaterials as potential scaffolds for tissue engineering. Philos Trans A Math Phys Eng Sci 2010;368:1981-97. 
20. Andreu V, Mendoza G, Arruebo M, Irusta S. Smart dressings based on nanostructured fibers containing natural origin antimicrobial, anti-inflammatory, and regenerative compounds. Materials (Basel) 2015;8:5154-93.

21. Fukushima S, Motoyama K, Tanida Y, Higashi T, Ishitsuka Y, Kondo $Y$, et al. Clinical evaluation of novel natural polysaccharides sacran as a skincare material for atopic dermatitis patients. J Cosmet Dermatological Sci Appl 2016;1:9-18.

22. Wathoni N, Motoyama K, Higashi T, Okajima M, Kaneko T, Arima H. Physically crosslinked-sacran hydrogel films for wound dressing application. Int J Biol Macromol 2016;89:465-70.

23. Wathoni N, Motoyama K, Higashi T, Okajima M, Kaneko T, Arima $H$. Enhancing effect of $\gamma$-cyclodextrin on wound dressing properties of sacran hydrogel film. Int J Biol Macromol 2017;94:181-6.

24. Wathoni N, Motoyama K, Higashi T, Okajima M, Kaneko T, Arima H. Enhancement of curcumin wound healing ability by complexation with 2-hydroxypropyl-g-cyclodextrin in sacran hydrogel film. Int J Biol Macromol 2017;98:268-76.

25. Koo H, Jin G, Kang H, Lee $Y$, Nam HY, Jang $H$, et al. A new biodegradable crosslinked polyethene oxide sulfide (PEOS) hydrogel for controlled drug release. Int J Pharm 2009;374:58-65.

26. Xu FJ, Kang ET, Neoh KG. pH-and temperature-responsive hydrogels from crosslinked triblock copolymers prepared via consecutive atom transfer radical polymerizations. Biomaterials 2006;27:2787-97.

27. Zhang Y, Zhang M, Jiang H, Shi J, Li F, Xia Y, et al. Bio-inspired layered chitosan/graphene oxide nanocomposite hydrogels with high strength and $\mathrm{pH}$-driven shape memory effect. Carbohydr Polym 2017;177:116-25.

28. Mateescu A, Wang Y, Dostalek J, Jonas U. Thin hydrogel films for optical biosensor applications. Membranes (Basel) 2012;2:40-69.

29. Luo Y, Kirker KR, Prestwich GD. Cross-linked hyaluronic acid hydrogel films: new biomaterials for drug delivery. J Controlled Release 2000;69:169-84.

30. Kamoun EA, Kenawy ERS, Chen X. A review on polymeric hydrogel membranes for wound dressing applications: PVAbased hydrogel dressings. J Adv Res 2017;8:217-33.

31. Pereira R, Carvalho A, Vaz DC, Gil MH, Mendes A, Bartolo P. Development of novel alginate-based hydrogel films for wound healing applications. Int J Biol Macromol 2013;52:221-30.

32. Kikumoto $Y$, Sugiyama $H$, Inoue $T$, Morinaga $H$, Takiue $K$, Kitagawa M, et al. Sensitization to alloxan-induced diabetes and pancreatic cell apoptosis in acatalasemic mice. Biochim Biophys Acta-Mol Basis Dis 2010;1802:240-6.

33. Jimenez PA, Rampy MA. Keratinocyte growth factor-2 accelerates wound healing in incisional wounds. J Surg Res 1999;81:238-42. 